\title{
On fluid motion in librating ellipsoids with moderate equatorial eccentricity
}

\author{
KEKE ZHANG ${ }^{1} \uparrow$, KIT H. CHAN ${ }^{2}$ AND XINHAO LIAO \\ ${ }^{1}$ Department of Mathematical Sciences, University of Exeter, Exeter EX4 4QF, UK \\ ${ }^{2}$ Department of Mathematics, University of Hong Kong, Pokfulam, Hong Kong \\ ${ }^{3}$ Shanghai Astronomical Observatory, Chinese Academy of Sciences, Shanghai 200030, China
}

(Received 12 December 2010; revised 4 February 2011; accepted 9 February 2011; first published online 15 March 2011)

The motion of a homogeneous fluid of viscosity $v$ confined in a librating ellipsoidal cavity with semi-axes $a$ and moderate equatorial eccentricity $\mathscr{E}$ is investigated. The ellipsoid rotates with an angular velocity $\Omega_{0}(1+\delta \sin \hat{\omega} t)$, where $\Omega_{0}$ is the mean rate of rotation, $\hat{\omega}$ is the libration frequency and $\Omega_{0} \delta$ represents the amplitude of longitudinal libration. When $\delta \ll \mathscr{E}^{2}$ and $E^{1 / 2} \ll \mathscr{E}^{2} \ll 1$, where $E$ is the Ekman number defined as $E=v /\left(a^{2} \Omega_{0}\right)$, an explicit analytical solution describing fluid motion in librating ellipsoids is derived for any size of the libration frequency $\hat{\omega}$. Threedimensional numerical simulations of the same problem are also performed, revealing the generation of mean zonal flow in librating ellipsoidal cavities and showing a satisfactory agreement between the asymptotic and numerical analyses.

Key words: geophysical and geological flows, rotating flows, topographic effects

\section{Introduction and formulation}

The combined effects of rotation and varying gravity cause most planets to assume an ellipsoidal shape (for example, Dermott 1979). Interaction between an ellipsoidal planet and its parent star or moon can force longitudinal libration of the planet, a periodic variation of its angular velocity around its rotating axis (for example, William et al. 2001; Margot et al. 2007; Noir et al. 2009). A number of authors have studied librationally driven flows in spherical cavities. Aldridge \& Toomre (1969) investigated experimentally librationally driven flows in a spherical container, revealing the resonance of inertial modes when the librating frequency is close to that of an inertial mode. Rieutord (1991) and Tilgner (1999) studied numerically the response to longitudinal libration in spherical systems (see also Calkins et al. 2010). In an experimental and numerical study, Noir et al. (2009) demonstrated that, depending on the libration amplitude, there exist a number of different classes of librationally driven flows. When the libration frequency is sufficiently small in comparison to the rotation rate, Busse (2010) showed that longitudinal libration can drive a steady zonal flow in librating spheres, which is confirmed by a recent experimental and numerical study (Sauret et al. 2010). In spherical geometry, coupling between a librating container and its interior fluid is purely viscous, through the effect of a thin viscous boundary layer at the bounding surface of the container. 
This paper represents the first asymptotic and numerical study that is concerned with the dynamic response of a homogeneous incompressible Newtonian fluid with kinematic viscosity $v$ confined within a ellipsoidal cavity with moderate equatorial eccentricity $\mathscr{E}$ to longitudinal libration. The ellipsoidal cavity is described by

$$
\frac{x^{2}}{a^{2}}+\frac{y^{2}}{a^{2}\left(1-\mathscr{E}^{2}\right)}+\frac{z^{2}}{a^{2}}=1
$$

and rotates with an angular velocity $\Omega=\hat{z}\left[\Omega_{0}(1+\delta \sin \hat{\omega} t)\right]$, where $\Omega_{0}$ is the mean rate of rotation, $\hat{z}$ is a unit vector in the direction of rotation axis, $\hat{\omega}$ is the libration frequency and $\Omega_{0} \delta$ represents the amplitude of libration. We shall focus on the case of moderate equatorial eccentricity $E^{1 / 2} \ll \mathscr{E}^{2} \ll 1$, where $E$ is the Ekman number defined as $E=v /\left(a^{2} \Omega_{0}\right)$, with the flux from the viscous boundary layer being of the order $E^{1 / 2}$ and the deformation from a sphere being of the order $\mathscr{E}^{2}$. Since typical values of the Ekman number $E$ for many librating planets and satellites (see table 2 in Noir et al. 2009) are extremely small, $E \leqslant O\left(10^{-14}\right)$, the topographic coupling between a librating mantle and its liquid core is usually predominant. For a librating ellipsoid with $E^{1 / 2} \ll \mathscr{E}^{2} \ll 1$, we expect that the viscous effect, in comparison to the topographic effect, is negligible in the leading-order approximation. This expectation is confirmed by the result of the corresponding numerical simulation that includes the full viscous effect.

In a frame of reference attached to the ellipsoidal container, the mantle frame, librationally driven flow is governed by the dimensionless equations of motion and continuity,

$$
\begin{gathered}
\frac{\partial \boldsymbol{u}}{\partial t}+\boldsymbol{u} \cdot \nabla \boldsymbol{u}+2 \hat{z} \times \boldsymbol{u}+\nabla p=E \nabla^{2} \boldsymbol{u}-2 \delta \hat{z} \times \boldsymbol{u} \sin \left(\hat{\omega}_{l} t\right)+\left(\delta \hat{\omega}_{l}\right)(\boldsymbol{r} \times \hat{z}) \cos \left(\hat{\omega}_{l} t\right), \\
\nabla \cdot \boldsymbol{u}=0,
\end{gathered}
$$

where $\hat{\omega}_{l}=\hat{\omega} / \Omega_{0}$ with $0<\left|\hat{\omega}_{l}\right|<2$ is the dimensionless frequency of libration, $\delta$ is often called the Poincare number which is usually small, $\boldsymbol{r}$ is the position vector, $p$ is a reduced pressure and $\boldsymbol{u}$ is the three-dimensional velocity field $\boldsymbol{u}=\left(u_{r}, u_{\theta}, u_{\phi}\right)$ with corresponding unit vectors $(\hat{\boldsymbol{r}}, \hat{\boldsymbol{\theta}}, \hat{\boldsymbol{\phi}})$ in spherical polar coordinates $(r, \theta, \phi)$ with $\theta=0$ at the axis of $\hat{z}$. The final term on the right-hand side of (1.2) is known as the Poincare force. Here we have employed $a$ as the length scale, $\Omega_{0}^{-1}$ as the unit of time and $\rho a^{2} \Omega_{0}^{2}$ as the unit of pressure. Librationally driven flow on the bounding surface, $\mathscr{S}$, of the ellipsoidal cavity is at rest, requiring that

$$
\hat{\boldsymbol{n}} \cdot \boldsymbol{u}=\hat{\boldsymbol{n}} \times \boldsymbol{u}=\mathbf{0},
$$

where $\hat{\boldsymbol{n}}$ denotes the normal to the ellipsoidal surface $\mathscr{S}$.

In what follows we shall present the asymptotic analysis of (1.2) and (1.3) for a librating ellipsoid with a small $E$ and moderate $\mathscr{E}$ in $\S 2$. The fully three-dimensional numerical simulation of the same problem, valid for any size of $E$ and $\mathscr{E}$, is discussed in $\S 3$ along with some remarks.

\section{Asymptotic solution for librating flows}

For weakly librating flows in an ellipsoidal cavity with moderate equatorial eccentricity marked by $\delta \ll \mathscr{E}^{2}$ and $E^{1 / 2} \ll \mathscr{E}^{2} \ll 1$, the viscous effect may be neglected in the first approximation and the librating flows are then governed 
by

$$
\begin{aligned}
\frac{\partial \boldsymbol{u}}{\partial t}+\boldsymbol{u} \cdot \nabla \boldsymbol{u}+2 \hat{z} \times \boldsymbol{u}+\nabla p & =-2 \delta \hat{z} \times \boldsymbol{u} \sin \left(\hat{\omega}_{l} t\right)+\left(\delta \hat{\omega}_{l}\right)(\boldsymbol{r} \times \hat{z}) \cos \left(\hat{\omega}_{l} t\right), \\
\nabla \cdot \boldsymbol{u} & =0,
\end{aligned}
$$

while the non-slip boundary condition (1.4) becomes

$$
\hat{\boldsymbol{n}} \cdot \boldsymbol{u}=0 .
$$

A solution to (2.1) and (2.2) can be sought by expanding the variables, $\boldsymbol{u}$ and $p$, in a double series in powers of $\delta$ and $\mathscr{E}^{2}$,

$$
\boldsymbol{u}=\delta \boldsymbol{u}_{0}+\left(\delta \mathscr{E}^{2}\right) \boldsymbol{u}_{1}+\cdots, \quad p=\delta p_{0}+\left(\delta \mathscr{E}^{2}\right) p_{1}+\cdots,
$$

where $\boldsymbol{u}_{0}$ and $p_{0}$ denote the leading-order solution directly driven by the Poincaré forcing while $\boldsymbol{u}_{1}$ represents the flow in connection with the topographic effect of an ellipsoidal container. For an ellipsoidal cavity with moderate equatorial eccentricity $E^{1 / 2} \ll \mathscr{E}^{2} \ll 1$, we may also expand the normal $\hat{\boldsymbol{n}}$ of the bounding ellipsoidal surface $\mathscr{S}$ around the spherical surface $r=1$, which gives rise to

$$
\hat{\boldsymbol{n}}=\hat{\boldsymbol{r}}+\frac{\mathscr{E}^{2}}{2}\left[\hat{\boldsymbol{r}} \sin ^{2} \theta \sin ^{2} \phi+\hat{\boldsymbol{\theta}} \sin 2 \theta \sin ^{2} \phi+\hat{\boldsymbol{\phi}} \sin \theta \sin 2 \phi\right]+\cdots .
$$

Note that harmonic terms like $\sin 4 \phi$ would appear at higher orders in the expansion (2.5), which are neglected in the present analysis.

After substitution of the expansion (2.4) into (2.1) and (2.2) and the expansion of $\hat{\boldsymbol{n}}$, given by (2.5), into the boundary condition (2.3), the leading-order problem in the asymptotic sequence is governed by the equations,

$$
\frac{\partial \boldsymbol{u}_{0}}{\partial t}+2 \hat{z} \times \boldsymbol{u}_{0}+\nabla p_{0}=\hat{\omega}_{l}(\boldsymbol{r} \times \hat{z}) \cos \left(\hat{\omega}_{l} t\right), \quad \nabla \cdot \boldsymbol{u}_{0}=0,
$$

which are subject to the leading-order boundary condition $\hat{\boldsymbol{r}} \cdot \boldsymbol{u}_{0}=0$. It can be readily shown that the leading-order solution in the double expansion (2.4) takes the form

$$
\boldsymbol{u}_{0}=-\left(r \sin \theta \sin \hat{\omega}_{l} t\right) \hat{\boldsymbol{\phi}}, \quad p_{0}=-(r \sin \theta)^{2} \sin \hat{\omega}_{l} t .
$$

This represents an oscillatory and axisymmetric zonal flow.

Topographic effects enter into the problem at the next order $\left(\delta \mathscr{E}^{2}\right)$. We first look at the boundary condition needed at this order. Substitution of the expansion of $\hat{\boldsymbol{n}}$ into (2.3) yields the condition at the spherical surface $r=1$ for $\boldsymbol{u}_{1}$,

$$
\left(\hat{\boldsymbol{r}} \cdot \boldsymbol{u}_{1}\right)_{r=1}=-\frac{1}{2} \boldsymbol{u}_{0} \cdot\left[\hat{\boldsymbol{r}} \sin ^{2} \theta \sin ^{2} \phi+\hat{\boldsymbol{\theta}} \sin 2 \theta \sin ^{2} \phi+\hat{\boldsymbol{\phi}} \sin \theta \sin 2 \phi\right] .
$$

In other words, the problem at the next order $\left(\delta \mathscr{E}^{2}\right)$ is governed by a homogeneous partial differential equation with an inhomogeneous boundary condition (2.8). It is usually mathematically convenient, however, to solve an inhomogeneous partial differential equation with a homogeneous boundary condition. This suggests that we may introduce a new variable,

$$
\boldsymbol{U}_{1}=\boldsymbol{u}_{1}-\frac{r^{2}}{2}\left[\hat{\boldsymbol{r}} \sin ^{2} \theta \sin 2 \phi+\hat{\boldsymbol{\phi}} 2 \sin ^{3} \theta \cos 2 \phi\right] \sin \hat{\omega}_{l} t,
$$

such that $\boldsymbol{U}_{1}$ satisfies both the homogeneous boundary condition at the spherical surface $r=1$ and the divergence-free condition. It follows that $\boldsymbol{U}_{1}$ is described by the equations, 


$$
\begin{array}{r}
\frac{\partial \boldsymbol{U}_{1}}{\partial t}+2 \hat{\boldsymbol{z}} \times \boldsymbol{U}_{1}+\nabla P_{1}=-\frac{r^{2} \hat{\omega}_{l} \sin ^{2} \theta}{2}[\hat{\boldsymbol{r}} \sin 2 \phi+\hat{\boldsymbol{\phi}} \sin \theta \cos 2 \phi] \cos \hat{\omega}_{l} t \\
+2 r^{2} \sin ^{3} \theta\left[\hat{\boldsymbol{r}} \sin \theta \cos 2 \phi+\hat{\boldsymbol{\theta}} \cos \theta \cos 2 \phi-\frac{\hat{\boldsymbol{\phi}}}{2} \sin 2 \phi\right] \sin \hat{\omega}_{l} t,
\end{array}
$$

$$
\nabla \cdot \boldsymbol{U}_{1}=0
$$

where $\boldsymbol{U}_{1}$ satisfies the homogeneous boundary condition

$$
\hat{\boldsymbol{r}} \cdot \boldsymbol{U}_{1}=0 \text { at } \boldsymbol{r}=1 .
$$

A mathematically convenient way of solving (2.10) and (2.11) with the boundary condition (2.12) is to assume that inertial modes, which automatically satisfy (2.11) and (2.12), form a complete function system (Zhang, Chan \& Liao 2010) and, thus, can be employed to represent an arbitrary profile of $\boldsymbol{U}_{1}$. In other words, the problem may be solved by making the following expansions:

$$
\begin{gathered}
\boldsymbol{U}_{1}=\sum_{n k}\left(A_{n k} \boldsymbol{u}_{n k} \mathrm{e}^{\mathrm{i} 2 \phi}+\text { c.c. }\right) \sin \hat{\omega}_{l} t+\sum_{n k}\left(B_{n k} \boldsymbol{u}_{n k} \mathrm{e}^{\mathrm{i} 2 \phi}+\text { c.c. }\right) \cos \hat{\omega}_{l} t, \\
P_{1}=\sum_{n k}\left(A_{n k} p_{n k} \mathrm{e}^{\mathrm{i} 2 \phi}+\text { c.c. }\right) \sin \hat{\omega}_{l} t+\sum_{n k}\left(B_{n k} p_{n k} \mathrm{e}^{\mathrm{i} 2 \phi}+\text { c.c. }\right) \cos \hat{\omega}_{l} t,
\end{gathered}
$$

where $\mathrm{i}=\sqrt{-1}, A_{n k}$ and $B_{n k}$ are complex coefficients to be determined, c.c. denotes the complex conjugate of the preceding term and $\boldsymbol{u}_{n k}(r, \theta) \mathrm{e}^{\mathrm{i} 2 \phi}$ and $p_{n k}(r, \theta) \mathrm{e}^{\mathrm{i} 2 \phi}$ satisfy the equations,

$$
\left(\mathrm{i} 2 \sigma_{n k}+2 \hat{z} \times\right)\left(\boldsymbol{u}_{n k} \mathrm{e}^{\mathrm{i} 2 \phi}\right)=-\nabla\left(p_{n k} \mathrm{e}^{\mathrm{i} 2 \phi}\right), \quad \nabla \cdot\left(\boldsymbol{u}_{n k} \mathrm{e}^{\mathrm{i} 2 \phi}\right)=0,
$$

where $\sigma_{n k}$ is the half frequency of a spherical inertial mode with $\left|\sigma_{n k}\right|<1$, subject to the condition $\hat{\boldsymbol{r}} \cdot \boldsymbol{u}_{n k}=0$ at $r=1$. The explicit general analytical solution for spherical inertial modes was found by Zhang et al. (2001). In the present analysis, $\boldsymbol{u}_{n k}(r, \theta)$ and $p_{n k}(r, \theta)$ for azimuthal wavenumber $m=2$ with the required symmetry can be extracted from the general solution, and are

$$
\begin{aligned}
p_{n k}= & \sum_{i=0}^{k} \sum_{j=0}^{k-i} C_{k i j} r^{2(1+i+j)} \sigma_{n k}^{2 i-1}\left(1-\sigma_{n k}^{2}\right)^{j-1} \sin ^{2+2 j} \theta \cos ^{2 i} \theta, \\
\hat{\boldsymbol{r}} \cdot \boldsymbol{u}_{n k}= & -\mathrm{i} \sum_{i=0}^{k} \sum_{j=0}^{k-i} C_{k i j} r^{1+2(i+j)} \sigma_{n k}^{2 i-1}\left(1-\sigma_{n k}^{2}\right)^{j-1} \\
& \times \sin ^{2+2 j} \theta \cos ^{2 i} \theta\left[\sigma_{n k}\left(1+\sigma_{n k}+j \sigma_{n k}\right)-i\left(1-\sigma_{n k}^{2}\right)\right], \\
\hat{\boldsymbol{\theta}} \cdot \boldsymbol{u}_{n k}= & -\mathrm{i} \sum_{i=0}^{k} \sum_{j=0}^{k-i} C_{k i j} r^{1+2(i+j)} \sigma_{n k}^{2 i-1}\left(1-\sigma_{n k}^{2}\right)^{j-1} \\
& \times \sin ^{1+2 j} \theta \cos ^{2 i-1} \theta\left[\sigma_{n k}\left(1+\sigma_{n k}+j \sigma_{n k}\right) \cos ^{2} \theta+i\left(1-\sigma_{n k}^{2}\right) \sin ^{2} \theta\right], \\
\hat{\boldsymbol{\phi}} \cdot \boldsymbol{u}_{n k}= & \sum_{i=0}^{k} \sum_{j=0}^{k-i} C_{k i j} r^{1+2(i+j)} \sin ^{1+2 j} \theta \cos ^{2 i} \theta \sigma_{n k}^{2 i}\left(1-\sigma_{n k}^{2}\right)^{j-1}\left(1+\sigma_{n k}+j\right),
\end{aligned}
$$


where the indices $(n, k)$ are related to the spatial wavenumbers in the vertical and radial directions, respectively, and $C_{k i j}$ is defined as

$$
C_{k i j}=\frac{(-1)^{i+j}[2(k+i+j)+3] ! !}{2^{j+1}(2 i-1) ! !(k-i-j) ! i ! j !(2+j) !},
$$

along with $(2 i-1) ! !=(2 i-1) \cdots(3)(1),(-1) ! !=1,(0) !=1$. The half frequencies of the spherical inertial modes in (2.16)-(2.19), $\sigma_{n k}$, are solutions of

$$
\begin{aligned}
0=\frac{[2(k+2)] !}{k !(k+2) !}+\sum_{j=0}^{k-1} \frac{(-1)^{j+k}[2(2 k+2-j)] !}{j !(2 k+2-j) ![2(k-j)] !} \\
\times\left[(1+k-j) \sigma_{n k}-2(k-j)\right] \sigma_{n k}^{2(k-j)-1},
\end{aligned}
$$

where $k=1,2,3 \ldots$. There exist $2 k$ different solutions to (2.21), which can be arranged according to the size of $\left|\sigma_{n k}\right|, 0<\left|\sigma_{1 k}\right|<\left|\sigma_{2 k}\right|<\left|\sigma_{3 k}\right|, \ldots,<\left|\sigma_{n k}\right|<\ldots$ In other words, $\sigma_{n k}$ denotes the $n$-th smallest root of (2.21) for given $k$. The two principal inertial modes, which have the simplest spatial structure, are characterized by frequencies $\omega_{n k}=2 \sigma_{n k}$ with $k=1$ and $n=1,2: \omega_{11}=2 \sigma_{11}=-0.2319$ and $\omega_{21}=2 \sigma_{21}=1.2319$.

The major task of our asymptotic analysis is to find the analytical expression for coefficients $A_{n k}$ and $B_{n k}$ in the expansions (2.13) and (2.14). After substituting the expansions into (2.10), multiplying the resulting equation by the complex conjugate of $\boldsymbol{u}_{n k} \mathrm{i}^{\mathrm{i} 2 \phi}$ and then integrating over the sphere, we obtain two equations for $A_{n k}$ and $B_{n k}$,

$$
\begin{aligned}
& A_{n k}\left(\omega_{n k}^{2}-\hat{\omega}_{l}^{2}\right) \int_{V}\left|\boldsymbol{u}_{n k}\right|^{2} \mathrm{~d} V=\left(\frac{\hat{\omega}_{l}^{2}-\omega_{n k}}{2}\right) \mathscr{I}_{1}+\mathrm{i} \omega_{n k} \mathscr{I}_{2}-\frac{\mathrm{i} \hat{\omega}_{l}^{2} \mathscr{I}_{3}}{4}+\mathrm{i} \omega_{n k} \mathscr{I}_{4}, \\
& B_{n k}\left(\omega_{n k}^{2}-\hat{\omega}_{l}^{2}\right) \int_{V}\left|\boldsymbol{u}_{n k}\right|^{2} \mathrm{~d} V=\hat{\omega}_{l}\left[\frac{\mathrm{i}\left(1-\omega_{n k}\right) \mathscr{I}_{1}}{2}+\mathscr{I}_{2}-\frac{\omega_{n k} \mathscr{I}_{3}}{4}+\mathscr{I}_{4}\right],
\end{aligned}
$$

where $k \geqslant 1,1 \leqslant n \leqslant 2 k, \int_{V}$ denotes the volume integration over the sphere, and $\mathscr{I}_{1}, \mathscr{I}_{2}, \mathscr{I}_{3}$ and $\mathscr{I}_{4}$ - representing the integrals resulting from the product of the complex conjugate of $\boldsymbol{u}_{n k} \mathrm{e}^{\mathrm{i} 2 \phi}$ and the right-hand side of (2.10) - are given by

$$
\begin{aligned}
\mathscr{I}_{1}= & 2 \pi \int_{0}^{\pi} \int_{0}^{1}\left(\hat{\boldsymbol{\phi}} \cdot \boldsymbol{u}_{n k}^{*}\right) r^{4} \sin ^{4} \theta \mathrm{d} r \mathrm{~d} \theta \\
= & \sum_{i=0}^{k} \sum_{j=0}^{k-i} \frac{C_{k i j} 2^{j+2}(j+2) !(2 i-1) ! !}{(i+j+3)(2 i+2 j+5) ! !}\left[\sigma_{n k}^{2 i}\left(1-\sigma_{n k}^{2}\right)^{j-1}\left(1+\sigma_{n k}+j\right)\right], \\
\mathscr{I}_{2}= & 2 \pi \int_{0}^{\pi} \int_{0}^{1}\left(\hat{\boldsymbol{\theta}} \cdot \boldsymbol{u}_{n k}^{*}\right) r^{4} \sin ^{4} \theta \cos \theta \mathrm{d} r \mathrm{~d} \theta \\
= & 2 \pi \mathrm{i} \sum_{i=0}^{k} \sum_{j=0}^{k-i} \frac{C_{k i j} 2^{j+2}(j+2) !(2 i-1) ! !}{(i+j+3)(2 i+2 j+7) ! !}\left[\sigma_{n k}^{2 i-1}\left(1-\sigma_{n k}^{2}\right)^{j-1}\right] \\
& \times\left[(2 i+1) \sigma_{n k}+(j-4 i+1) \sigma_{n k}^{2}+2 i(j+3)\right] \\
\mathscr{I}_{3}= & 2 \pi \int_{0}^{\pi} \int_{0}^{1}\left(\hat{\boldsymbol{r}} \cdot \boldsymbol{u}_{n k}^{*}\right) r^{4} \sin ^{3} \theta \mathrm{d} r \mathrm{~d} \theta \\
= & 2 \pi \mathrm{i} \sum_{i=0}^{k} \sum_{j=0}^{k-i} \frac{C_{k i j} 2^{j+2}(j+2) !(2 i-1) ! !}{(i+j+3)(2 i+2 j+5) ! !}\left[\sigma_{n k}^{2 i-1}\left(1-\sigma_{n k}^{2}\right)^{j-1}\right] \\
& \times\left[\sigma_{n k}\left(1+\sigma_{n k}+j \sigma_{n k}\right)-i\left(1-\sigma_{n k}^{2}\right)\right],
\end{aligned}
$$




$$
\begin{aligned}
\mathscr{I}_{4}= & 2 \pi \int_{0}^{\pi} \int_{0}^{1}\left(\hat{\boldsymbol{r}} \cdot \boldsymbol{u}_{n k}^{*}\right) r^{4} \sin ^{5} \theta \mathrm{d} r \mathrm{~d} \theta \\
= & 2 \pi \mathrm{i} \sum_{i=0}^{k} \sum_{j=0}^{k-i} \frac{C_{k i j} 2^{j+3}(j+3) !(2 i-1) ! !}{(i+j+3)(2 i+2 j+7) ! !}\left[\sigma_{n k}^{2 i-1}\left(1-\sigma_{n k}^{2}\right)^{j-1}\right] \\
& \times\left[\sigma_{n k}\left(1+\sigma_{n k}+j \sigma_{n k}\right)-i\left(1-\sigma_{n k}^{2}\right)\right]
\end{aligned}
$$

where the half frequency of an inertial mode, $\sigma_{n k}$, can be readily obtained from (2.21) and $C_{k i j}$ is given by (2.20).

At first glance, (2.22) and (2.23) indicate that resonance with inertial modes would occur when the libration frequency $\hat{\omega}_{l}$ approaches the frequency of an inertial mode $\omega_{n k}:\left|A_{n k}\right| \rightarrow \infty$ and $\left|B_{n k}\right| \rightarrow \infty$ as $\hat{\omega}_{l} \rightarrow \omega_{n k}$. An essential step in the analysis is then to evaluate the complicated integrals on the right-hand side of (2.22) and (2.23). We first consider the right-hand side of (2.23) to determine the values of $B_{n k}$. With the expressions for $\mathscr{I}_{1}, \mathscr{I}_{2}, \mathscr{I}_{3}$ and $\mathscr{I}_{4}$ given by (2.24)-(2.27) together with $C_{k i j}$ given by (2.20), we can show, after some straightforward but cumbersome manipulation, that

$$
\begin{aligned}
\tilde{\mathscr{B}}_{n k} & =\left(\frac{B_{n k}}{2 \pi \mathrm{i} \hat{\omega}_{l}}\right)\left(\omega_{n k}^{2}-\hat{\omega}_{l}^{2}\right) \int_{V}\left|\boldsymbol{u}_{n k}\right|^{2} \mathrm{~d} V \\
& =-\mathrm{i}\left[\frac{\mathrm{i}\left(1-\omega_{n k}\right) \mathscr{I}_{1}}{2}+\mathscr{I}_{2}-\frac{\omega_{n k} \mathscr{I}_{3}}{4}+\mathscr{I}_{4}\right] \\
& =\sum_{i=0}^{k} \sum_{j=0}^{k-i} \frac{(-1)^{i+j}[2(k+i+j)+3] ! !}{(k-i-j) ! i ! j ![2(i+j)+5] ! !}\left[\sigma_{n k}^{2 i}\left(1-\sigma_{n k}^{2}\right)^{j}\right] .
\end{aligned}
$$

It is, however, both surprising and remarkable that $\tilde{\mathscr{B}}_{n k} \equiv 0$, i.e. the complicated summation in (2.28) vanishes identically for all possible values of $n$ and $k$. Since this mathematical property is not only non-trivial and totally unexpected but also at the heart of determining the key physical properties of a librating flow in an ellipsoid, we shall outline a brief proof below.

First, we notice that the two indices $(i, j)$ in the summation (2.28) are so intimately entangled that a direct summation even for moderate $k$ is impossible, although the summation at $k=1$ can be easily carried out. A crucial step towards proving that $\tilde{\mathscr{B}}_{n k} \equiv 0$ for all possible values of $n$ and $k$ is to establish a recurrence relationship that links the large $k$ summations of (2.28) with the small $k$ ones. For this purpose, we introduce one additional index, $M$, by considering a new summation

$$
\tilde{\mathscr{B}}_{n k}^{M}=\sum_{i=0}^{k-M} \sum_{j=0}^{k-i-M} \frac{(-1)^{i+j}[2(k+i+j)+3] ! ! \sigma_{n k}^{2 i}\left(1-\sigma_{n k}^{2}\right)^{j}}{(k-i-j-M) ! i ! j ![2(i+j+M)+5] ! !}
$$

where $0 \leqslant M \leqslant(k-1)$ and, obviously, $\tilde{\mathscr{B}}_{n k}^{0}=\tilde{\mathscr{B}}_{n k}$. Even though the new summation (2.29) appears to be more complicated than the original (2.28), it can be shown or verified that $\tilde{\mathscr{B}}_{n k}^{M}$ obeys the following recurrence relationship:

$$
\tilde{\mathscr{B}}_{n k}^{M}=\left[\frac{2(M-k+1)}{k-M}\right] \tilde{\mathscr{B}}_{n k}^{M+1},
$$


which implies that

$$
\tilde{\mathscr{B}}_{n k}^{0}=\left[\frac{-2(k-1)}{k}\right] \tilde{\mathscr{B}}_{n k}^{1}=\left[\frac{2^{2}(k-2)}{k}\right] \tilde{\mathscr{B}}_{n k}^{2}=\cdots=\left[\frac{(-2)^{k-1}}{k}\right] \tilde{\mathscr{B}}_{n k}^{k-1} .
$$

At $M=k-1$, the summation (2.29) can be readily carried out, which gives rise to

$$
\tilde{\mathscr{B}}_{n k}^{k-1}=\sum_{i=0}^{1} \sum_{j=0}^{1-i} \frac{(-1)^{i+j} \sigma_{n k}^{2 i}\left(1-\sigma_{n k}^{2}\right)^{j}}{(1-i-j) ! i ! j !} \equiv 0 .
$$

By virtue of the recurrence relationship (2.31) and the summation (2.32) at $M=k-1$, we derive that

$$
B_{n k}=\tilde{\mathscr{B}}_{n k}^{0}=\tilde{\mathscr{B}}_{n k}^{k-1} \equiv 0
$$

for any possible values of $n$ and $k$. Moreover, the vanishing right-hand side of (2.23) also implies that

$$
\left(\mathscr{I}_{1}-\frac{\mathrm{i} \mathscr{I}_{3}}{2}\right)=\frac{2}{\omega_{n k}}\left(\frac{\mathscr{I}_{1}}{2}-\mathrm{i} \mathscr{I}_{2}-\mathrm{i} \mathscr{I}_{4}\right) .
$$

Making use of the relationship (2.34), we can derive, from (2.22), that

$$
A_{n k}\left(\omega_{n k}^{2}-\hat{\omega}_{l}^{2}\right) \omega_{n k} \int_{V}\left|\boldsymbol{u}_{n k}\right|^{2} \mathrm{~d} V=\left(\omega_{n k}^{2}-\hat{\omega}_{l}^{2}\right)\left(-\frac{\mathscr{I}_{1}}{2}+\mathrm{i} \mathscr{I}_{2}+\mathrm{i} \mathscr{I}_{4}\right) \text {. }
$$

The appearance of the factor $\left(\omega_{n k}^{2}-\hat{\omega}_{l}^{2}\right)$ on both sides of the above equation removes the possibility of resonance with any inertial mode in a librating ellipsoid. With the explicit expressions for $\mathscr{I}_{1}, \mathscr{I}_{2}, \mathscr{I}_{4}$ given by (2.24), (2.25) and (2.27), we obtain from (2.35) that

$$
\begin{aligned}
A_{n k}=\pi\left[\int_{V}\left|\boldsymbol{u}_{n k}\right|^{2} \mathrm{~d} V\right]^{-1} \sum_{i=0}^{k} \sum_{j=0}^{k-i} \sigma_{n k}^{2 i-1}\left(1-\sigma_{n k}^{2}\right)^{j-1} \\
\quad \times \frac{(-1)^{i+j+1}[2(k+i+j)+3] ! !\left[3+j+\sigma_{n k}(3+2 j)\right]}{(k-i-j) ! i ! j ![2(i+j)+5] ! !(i+j+3)},
\end{aligned}
$$

where carrying out the integration of $\left|\boldsymbol{u}_{n k}\right|^{2}$ over the sphere yields

$$
\begin{aligned}
\int_{V}\left|\boldsymbol{u}_{n k}\right|^{2} \mathrm{~d} V= & \sum_{i=0}^{k} \sum_{j=0}^{k-i} \sum_{q=0}^{k} \sum_{l=0}^{k-q} \frac{(-1)^{i+j+q+l} \pi(j+l+1) ! \sigma_{n k}^{2(i+q)}}{2[2(i+j+q+l)+5] ! !(2 i-1) ! !} \\
& \times\left\{\frac{8 i q(2 i+2 q-3) ! !(j+l+2)}{\sigma_{n k}^{2}}+\frac{4(2 i+2 q-1) ! !}{\left(1-\sigma_{n k}^{2}\right)^{2}}\right. \\
& \left.\times\left[\left(\sigma_{n k}+1+j \sigma_{n k}\right)\left(\sigma_{n k}+1+l \sigma_{n k}\right)+\left(\sigma_{n k}+1+j\right)\left(\sigma_{n k}+1+l\right)\right]\right\} \\
& \times\left[\frac{\left(1-\sigma_{n k}^{2}\right)^{j+l}[2(k+i+j)+3] ! ![2(k+q+l)+3] ! !}{(2 q-1) ! !(k-i-j) !(k-q-l) ! i ! q ! j ! l !(j+2) !(2+l) !}\right] .
\end{aligned}
$$

The six largest coefficients $\bar{A}_{n k}$, which are scaled by $\bar{A}_{n k}=A_{n k}\left[\int_{V}\left|\boldsymbol{u}_{n k}\right|^{2} \mathrm{~d} V\right]^{1 / 2}$, are shown in table 1. Furthermore, upon realizing that the nonlinear equation at the order $\delta^{2}$ is automatically satisfied because of

$$
\boldsymbol{u}_{0} \cdot \nabla \boldsymbol{u}_{0}+2 \hat{z} \times \boldsymbol{u}_{0} \sin \hat{\omega}_{l} t=\frac{1}{2} \nabla\left[(r \sin \theta)^{2} \sin ^{2}\left(\hat{\omega}_{l} t\right)\right]
$$




$\begin{array}{rrrc}n & k & \omega_{k n}=2 \sigma_{k n} & \left|\bar{A}_{k n}\right| \\ 1 & 1 & -0.231925 & 0.403325 \\ 2 & 1 & 1.231925 & 0.095736 \\ 1 & 2 & -0.101790 & 0.037458 \\ 3 & 2 & -1.092568 & 0.026989 \\ 4 & 2 & 1.643443 & 0.018030 \\ 1 & 3 & -0.057710 & 0.009087\end{array}$

TABLE 1. Six largest scaled coefficients $\bar{A}_{n k}$ computed from the analytical expression (2.36).

the fluid motion $\boldsymbol{u}$, up to the order $\left(\delta^{2} \mathscr{E}^{2}\right)$ and valid for any value of $\hat{\omega}_{l}$, can be expressed as

$$
\begin{aligned}
\boldsymbol{u}=\{-\delta(r \sin \theta) \hat{\boldsymbol{\phi}} & +\frac{\left(\delta \mathscr{E}^{2}\right) r^{2}}{2}\left[\hat{\boldsymbol{r}} \sin ^{2} \theta \sin 2 \phi+\hat{\boldsymbol{\phi}} 2 \sin ^{3} \theta \cos 2 \phi\right] \\
& \left.+\left(\delta \mathscr{E}^{2}\right)\left[\sum_{n k}\left(A_{n k} \boldsymbol{u}_{n k} \mathrm{e}^{\mathrm{i} 2 \phi}+\text { c.c. }\right)\right]\right\} \sin \hat{\omega}_{l} t+O\left(\delta^{2} \mathscr{E}^{2}\right),
\end{aligned}
$$

where the explicit expression for $A_{n k}$ is given by (2.36) (see table 1) while the explicit expression for $\boldsymbol{u}_{n k}$ is given by (2.17)-(2.19). While the validity of the asymptotic solution (2.39) is restricted by $E^{1 / 2} \ll \mathscr{E}^{2} \ll 1$, it is, because of the property (2.38), not restricted by $\delta \ll \mathscr{E}^{2}$.

In the asymptotic solution (2.39), the first term represents the axisymmetric flow while the second and third terms describe the non-axisymmetric flow with the azimuthal wavenumber $m=2$ in connection with the topographic effect of ellipsoidal geometry. Table 1 indicates that the six dominant inertial modes in (2.39) would lead to a reasonably accurate solution of the problem.

\section{Numerical simulation and some remarks}

In the three-dimensional numerical simulation, the fully nonlinear equations (1.2) and (1.3) are solved subject to the non-slip boundary condition (1.4). The primary purpose of our numerical analysis is to validate the key features of the asymptotic solution (2.39) in a librating ellipsoidal cavity with moderate equatorial eccentricity characterized by $E^{1 / 2} \ll \mathscr{E}^{2} \ll 1$ and to examine the effect of viscosity and nonlinearity neglected in the asymptotic analysis.

For simulating librating flows in non-spherical geometry such as an ellipsoid with moderate equatorial eccentricity, finite element methods - which are usually complicated and cumbersome in their numerical implementation - are particularly suitable. For example, a three-dimensional finite element mesh is flexible enough to construct more nodes in the vicinity of the bounding surface of an ellipsoid even with large equatorial eccentricity, for the purpose of resolving the thin viscous boundary layer. A sketch of the finite element mesh used in our numerical simulation is illustrated in figure 1. In our numerical simulation, a semi-implicit time stepping scheme is employed for the time advancement, a second-order extrapolation is applied to the nonlinear term and no spatial symmetries with respect to the equator or any other plane are imposed.

There are two obvious features emerging from the asymptotic solution (2.39): the longitudinal libration in ellipsoids cannot resonate with any inertial modes and, the weakly librating flows are, when re-scaled by $t_{\text {scaled }}=t \hat{\omega}_{l} /(2 \pi)$, independent of 


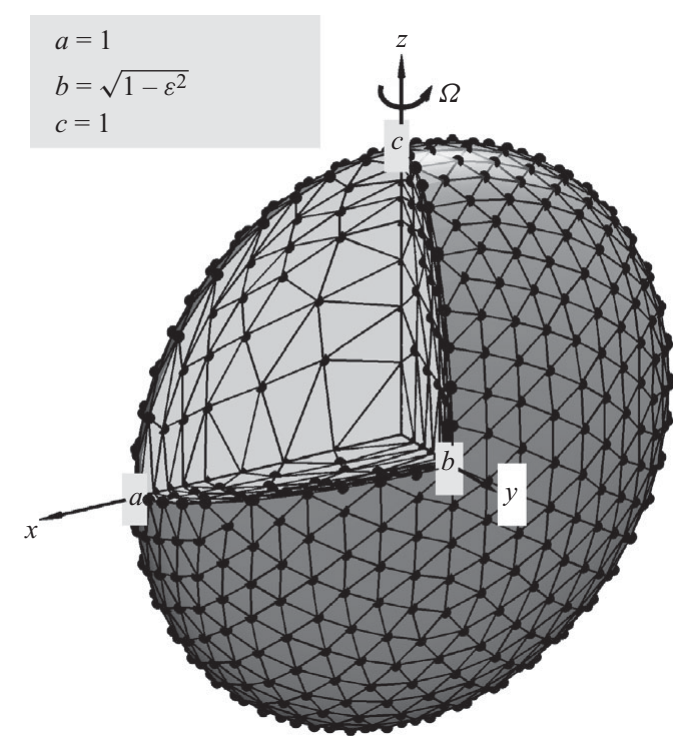

FIGURE 1. A schematic of the ellipsoidal meshes for numerical simulation with the denser mesh in the vicinity of its bounding surface.
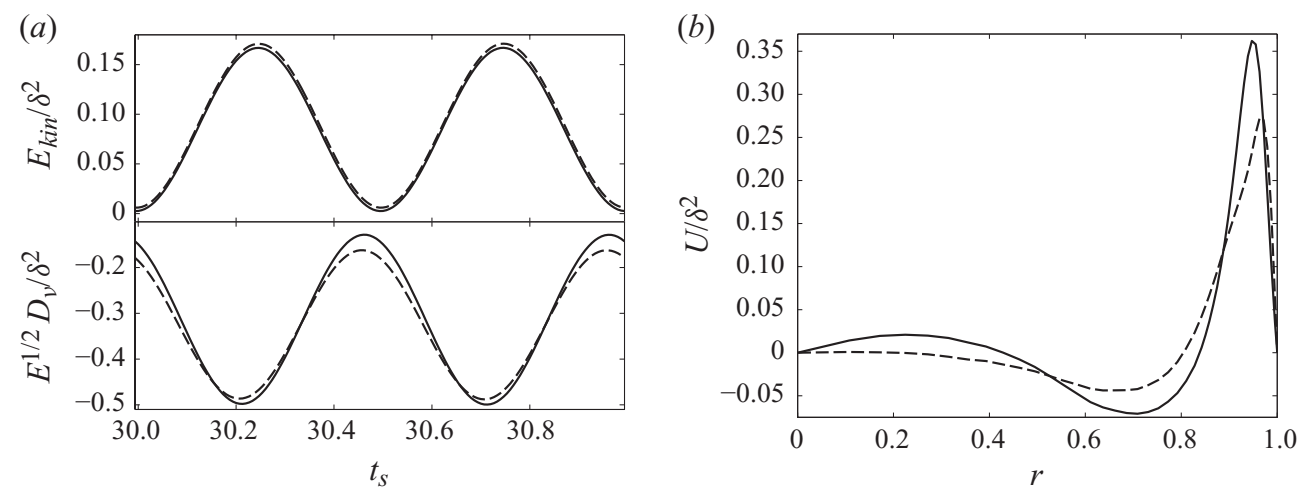

FIGURE 2. (a) Kinetic energy $E_{k i n}$ and viscous dissipation $D_{v}$ of the librationally driven flows plotted as a function of $t / T$, where $T=2 \pi / \hat{\omega}_{l}$ and $(b)$ the mean zonal flow, $U(r, \theta=\pi / 2)$, as a function of $r$ for $\delta=0.01, E=10^{-4}$ and $\mathscr{E}=0.5$ with $\hat{\omega}_{l}=1.0$ (solid line) and $\hat{\omega}_{l}=1.2319$ (dashed line).

the size of the libration frequency $\hat{\omega}_{l}$. Our numerical simulation will be also restricted to the regime $E^{1 / 2} \ll \mathscr{E}^{2} \ll 1$, consistent with that for the asymptotic solution (2.39). We have performed a large number of numerical simulations at many different values of $\hat{\omega}_{l}$ for a small Poincaré number $\delta=0.01$ and a small Ekman number $E=10^{-4}$ together with a moderate equatorial eccentricity $\mathscr{E}=0.5$. The result of two typical simulations is displayed in figure 2 , where $\hat{\omega}_{l}=1.2319$ is at the resonance frequency of an inertial mode with azimuthal wavenumber $m=2$ while $\hat{\omega}_{l}=1.0$ is not. It is worth noting that, although the scaled time $t_{\text {scaled }}=t \hat{\omega}_{l} /(2 \pi)$ is adopted in plotting the time dependence of numerical simulations, our actual computation always uses the unscaled time $t$. It can be seen that there is an expected agreement between the asymptotic solution and direct numerical simulation: the kinetic energy $E_{k i n}$ in figure 2 is quantitatively consistent with solution (2.39), resonance of any type cannot occur 
(a)

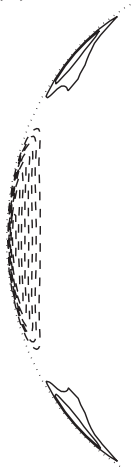

(c)
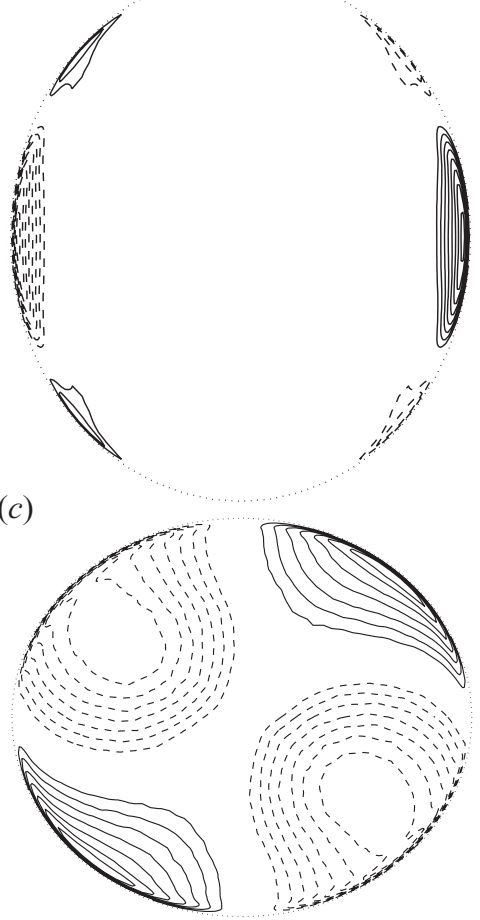

(b)

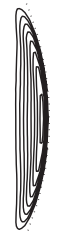

$(d)$
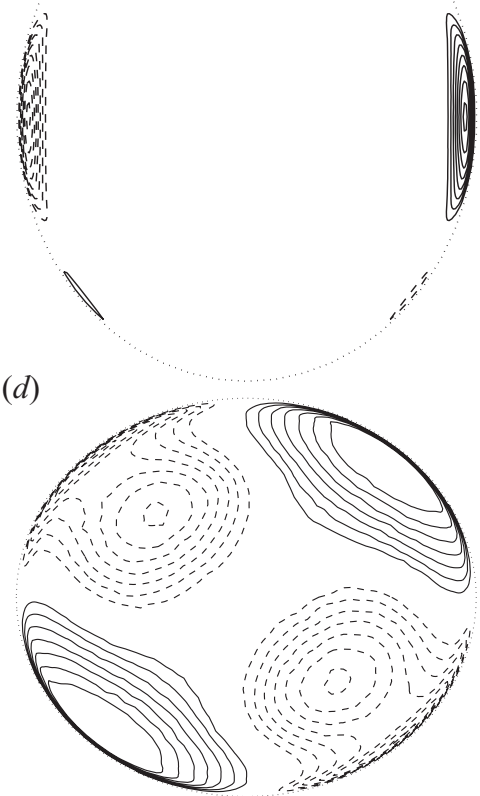

Figure 3. (a) Contours of the mean zonal flow $U(r, \theta)$ in the meridional plane at $x=0$ for $\omega_{l}=1.2319,(c)$ contours of the radial flow $u_{r}$ at the equatorial $z=0$ plane for $\omega_{l}=1.2319$, (b) contours of $U(r, \theta)$ in the meridional plane at $x=0$ for $\omega_{l}=1.0$ and $(d)$ contours of the radial flow $u_{r}$ at the equatorial $z=0$ plane for $\omega_{l}=1.0$. Solid contours are for $U>0\left(u_{r}>0\right)$ while dashed contours for $U<0\left(u_{r}<0\right)$. Other parameters of the two solutions are $\delta=0.01$ and $E=10^{-4}$ with $\mathscr{E}=0.5$.

in a librating ellipsoid with a moderate equatorial eccentricity even in the presence of viscous effects and, furthermore, the size of the libration frequency $\hat{\omega}_{l}$ plays a secondary role in determining the main properties of librating flows. Since the kinetic energy $E_{k i n}$ in this weakly nonlinear solution is dominated by the leading-order term shown in (2.39), there are no noticeable differences between the kinetic energies of two numerical solutions obtained at different libration frequencies, as predicted by the asymptotic solution. But the structure of the viscous boundary layer (where the viscous dissipation mainly takes place) at $\omega_{l}=1.2319$ would be different from that at $\omega_{l}=1.0$. This is reflected in noticeable differences, shown in figure $2(a)$, between the viscous dissipation $D_{v}$ - which is defined as $-[1 /(2 V)] \int_{V}|\nabla \times \boldsymbol{u}|^{2} \mathrm{~d} V$ with $V$ being the volume of the ellipsoidal cavity - of two numerical solutions with different frequencies. Figure 3(c,d) shows the structure of the radial flow $u_{r}$ at the equatorial $z=0$ plane, which, apart from the expected influence of the viscous boundary layer, exhibits the largely same structure as that of the inertial mode $\boldsymbol{u}_{11}$ (Zhang et al. 2001), in a good agreement with the asymptotic expression (2.39).

An important feature, as a result of the combined effect of small viscosity and the boundary-layer nonlinearity, is the generation of a mean zonal flow $U(r, \theta)$ (obtained by averaging over both time $t$ and the azimuthal angle $\phi$ of spheroidal polar coordinates) in a librating ellipsoidal cavity, which is shown in figure $2(b)$ for $E=10^{-4}$ and $\delta=0.01$ with two different frequencies $\omega_{l}=1.2319$ and $\omega_{l}=1.0$. In 
spherical geometry, where there exists the only viscous coupling between the librating container and fluid that produces only an axisymmetric flow, it has been found that a mean zonal flow $U(r, \theta)$ can be generated via the effect of the nonlinear spherical viscous boundary layer (Busse 2010; Sauret et al. 2010). In an ellipsoidal cavity, where the librational flow is non-axisymmetric and the coupling between the container and fluid is dominated by topographic effects, our simulation reveals that a mean zonal flow $U(r, \theta)$ can be also generated. The mechanism of maintaining the mean flow $U(r, \theta)$ in an ellipsoidal cavity would be similar to that in a librating sphere discussed by Busse (2010). As required by conservation of mass, the interior fluid will be sucked or ejected into the viscous boundary layer. Through this viscous process together with the nonlinear effect, the boundary layer communicates with the bulk of the fluid and maintains the time-independent equilibrium of the mean zonal flow $U(r, \theta)$. The two-dimensional profile for the mean zonal flow $U(r, \theta)$ at the meridional plane $x=0$ is displayed in figure $3(a, b)$ : slightly different profiles are likely to be attributable to changing structures of the viscous boundary layer at different libration frequencies.

Both the asymptotic and numerical solutions discussed in this paper are only for weakly librating flows with small Poincaré number $\delta \ll 1$. When the Poincare number is sufficiently large, the nonlinear effect would become strong and dominant. It is anticipated that there would be substantial differences between the prograde and retrograde phases and that the main features of the librating flow would depend on the size of the libration frequency. Direct numerical simulation for large Poincare numbers with different equatorial eccentricities is currently underway and will be reported in a future paper.

Finally, it is worth mentioning that the original purpose of our asymptotic and numerical analyses is to search for possible resonance with inertial modes in ellipsoidal geometry driven by longitudinal libration. We have reached, however, a negative conclusion: even if both the libration frequency and the azimuthal wavenumber of topographic forcing are exactly the same as those of non-axisymmetric inertial modes, longitudinal libration cannot resonate with any inertial modes in ellipsoidal geometry. Since the parameter regime $E^{1 / 2} \ll \mathscr{E}^{2} \ll 1$ is not only geophysically relevant but also accessible to laboratory experiments, it is hoped that the findings of this work will be validated by the ongoing experiments currently taking place in several laboratories.

K.Z. would like to thank J. Laskar who was able to provide an alternative proof for $\tilde{\mathscr{B}}_{n k}=0$ after K.Z. gave a talk on the problem. We also thank J. Noir and J. Aurnou for helpful discussion about the problem. K.Z. is supported by UK NERC and STFC grants, K.H.C. is supported by Hong Kong RGC grants 700308 and 700310 and X.L. is supported by NSFC/10633030 and CAS grants. The parallel computation is supported by Shanghai Supercomputer Center and Swiss National Supercomputing Center.

\section{REFERENCES}

Aldridge, K. D. \& Toomre, A. 1969 Axisymmetric inertial oscillations of a fluid in a rotating spherical container. J. Fluid Mech. 37, 307-323.

Busse, F. H. 2010 Mean zonal flows generated by librations of a rotating spherical cavity. J. Fluid Mech. 650, 505-512.

Calkins, M. A., Noir, J., Eldredge, J. \& Aurnou, J. M. 2010 Axisymmetric simulations of libration-driven fluid dynamics in a spherical shell geometry. Phys. Fluids 22, 086602.

Dermott, S. F. 1979 Shapes and gravitational moments of satellites and asteroids. Icarus 37, $576-586$. 
Margot, J. L., Peale, S. J., Jurgens., R. F., Slade, M. A. \& Holin, I. V. 2007 Large longitude libration of mercury reveals a molten core. Science 316, 710-714.

Noir, J., Hemmerlin, F., Wicht, J., Baca, S. M. \& Aurnou, J. M. 2009 An experimental and numerical study of librationally driven flow in planetary cores and subsurface oceans. Phys. Earth Planet. Inter. 173, 141-152.

RIEUTORD, M. 1991 Linear theory of rotating fluids using spherical harmonics. Part II. Time-periodic flows. Geophys. Astrophys. Fluid Dyn. 59, 185-208

Sauret, A., Cebron, D., Morize, C. \& Le Bars, M. 2010 Experimental and numerical study of mean zonal flows generated by librations of a rotating spherical cavity. J. Fluid Mech. 662, 260-268.

Tilgner, A. 1999. Driven inertial oscillations in spherical shells. Phys. Rev. E 59, 1789-1794.

William, J. G., Boggs, D. H., Yoder, C. F., Ratcliff, J. T. \& Dickey, J. O. 2001 Lunar rotational dissipation in solid body and molten core. J. Geophys. Res. 106, 27933-27968.

Zhang, K., Earnshaw, P., LiaO, X. \& Busse, F. H. 2001 On inertial waves in a rotating fluid sphere. J. Fluid Mech. 437, 103-119.

Zhang, K., Chan, K. \& LiaO, X. 2010 On fluid flows in precessing spheres in the mantle frame of reference. Phys. Fluid 22, 116604. 